\title{
Modelo de regressão por superfície de resposta como subsídio à estimativa de produtividade biológica de aveia para a produção de feno e silagem
}

\author{
Vanessa Pansera ${ }^{1}$ \\ DCEENG/UNIJUí, Ijuí, RS \\ Rúbia Diana Mantai ${ }^{2}$ \\ DCEENG/UNIJUí, Ijuí, RS \\ José Antonio Gonzalez da Silva ${ }^{3}$ \\ DEAG/UNIJUí, Ijuí, RS \\ Odenis Alessi ${ }^{4}$ \\ DCEENG/UNIJUÍ, Ijuí, RS \\ Adriana Roselia Kraisig 5 \\ DCEENG/UNIJUí, Ijuí, RS \\ Márcia Sostmeyer Jung ${ }^{6}$ \\ DEAG/UNIJUí, Ijuí, RS \\ Cibele Luisa Peter ${ }^{7}$ \\ DCEENG/UNIJUí, Ijuí, RS \\ Leonardo Norbert ${ }^{8}$ \\ DEAG/UNIJUÍ, Ijuí, RS
}

Resumo. Modelos de regressão são uma importante ferramenta para a simulação de processos agrícolas. A aveia é uma planta com alto potencial nutritivo e pode ter sua biomassa utilizada na alimentação animal. A produtividade biológica $(\mathrm{PB})$ da aveia é influenciada pela aplicação de nitrogênio e pelo momento de corte de biomassa, assim como, pelas condições meteorológicas, o que dificulta sua previsibilidade. O objetivo deste estudo é empregar modelos de regressão por superfície de resposta na simulação da produtividade biológica de aveia em função da dose de nitrogênio e do momento de corte de biomassa, para a produção de feno e silagem à alimentação animal, considerando as condições de ano agrícola. Para a realização do estudo, foi desenvolvido um experimento em condições reais de cultivo da aveia em seis anos agrícolas (2011 a 2016), com delineamento de blocos casualizados com 4 repetições em um fatorial $5 \times 4$ ( 5 doses de nitrogênio e 4 momentos de corte de biomassa). Foram obtidos modelos para condições de ano favoráveis, intermediárias e desfavoráveis à PB, além de um modelo geral independente de condição de ano. Os modelos apresentaram bons resultados de simulação, com enfoque para a produção de feno e silagem, quando consideradas práticas normalmente adotadas por produtores rurais. Assim, os modelos de regressão por superfície de resposta demonstram eficiência na simulação de PB da aveia

\footnotetext{
${ }^{1}$ Bolsista Capes/Brasil, vpansera@hotmail.com

${ }^{2}$ rdmantai@yahoo.com.br

3 jagsfaem@yahoo.com.br

4 odenisalessi@hotmail.com

${ }^{5}$ maryshelei@yahoo.com.br

${ }^{6}$ sostmeyerjungm@gmail.com

${ }^{7}$ cibele.peter2017@gmail.com

${ }^{8}$ norbert.leonardo6@gmail.com
} 
em função da dose de nitrogênio e momento de corte de biomassa, com enfoque na produção de feno e silagem, considerando a influência dos fatores meteorológicos dos anos agrícolas.

Palavras-chave. Modelos Estatísticos, Modelagem Matemática, Simulação, Alimentação Animal, Avena sativa

\section{Introdução}

Modelos de regressão por superfície de resposta constituem uma importante técnica de modelagem matemática. Através destes modelos é possível relacionar uma variável dependente a duas ou mais variáveis independentes, possibilitando a simulação e otimização de fenômenos reais. Na agricultura, os modelos de regressão são uma ferramenta útil à descrição de processos em plantas $[1,4]$.

A aveia é um cereal com diversos propósitos de uso e dentre eles destaca-se a utilização de sua biomassa para a alimentação animal, na forma de feno e silagem, por possuir alto valor nutritivo, com presença de proteína e outros nutrientes $[6,8]$. Um insumo agrícola que influencia diretamente na produção biológica desta planta é o nitrogênio (N). Este nutriente, quando aplicado em condições meteorológicas adequadas, estimula a produção de afilhos, ou seja, emissão de colmos da planta. Entretanto, é preciso atenção na quantidade fornecida, pois se houver aplicação inferior à necessária pode haver prejuízos para a planta, dificultando o desenvolvimento potencial da mesma. Por outro lado, se aplicado em demasia, o N pode acarretar no acamamento da aveia, reduzindo a qualidade da produção biológica e dificultando a colheita, além de causar poluição ambiental pelo excesso de N. Outro fator que interfere na produção biológica da aveia é o momento do corte de biomassa, normalmente realizado aos 90 dias após a emergência (DAE) para a produção de feno e aos 120 DAE para a produção de silagem. Entretanto, além da influência dos manejos citados, a produtividade biológica também é fortemente afetada pelos fatores meteorológicos, tanto nos efeitos diretos à planta, como na interferência da absorção de N, o que dificulta a previsibilidade sobre a produção de biomassa $[2,7,8]$.

O uso de regressões pode auxiliar na análise e simulação da produtividade biológica de aveia em diferentes condições meteorológicas em situações reais de cultivo. O objetivo deste estudo é empregar modelos de regressão por superfície de resposta na simulação da produtividade biológica de aveia em função da dose de nitrogênio aplicada e do momento de corte de biomassa, para a produção de feno e silagem à alimentação animal, considerando as condições de ano agrícola.

\section{Metodologia}

Para o desenvolvimento do estudo, foi realizado um experimento em condições reais de cultivo da aveia no Instituto Regional de Desenvolvimento Rural (IRDeR) pertencente à Universidade Regional do Noroeste do Estado do Rio Grande do Sul (UNIJUÍ) em Augusto Pestana (RS) em seis anos agrícolas (2011 a 2016). Foram utilizadas duas cultivares de aveia branca (Brisasul e Barbarasul), sendo consideradas as médias de seus resultados. O delineamento experimental empregado foi de blocos casualizados com quatro repetições, em um esquema fatorial $5 \times 4$, com 5

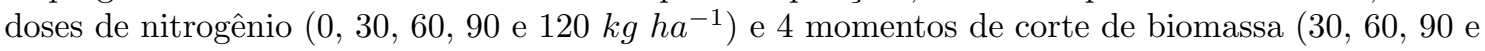
120 DAE), no sistema de sucessão soja/aveia. As parcelas experimentais foram constituídas de 5 $m^{2}$ e posteriormente os dados foram transformados para hectare. A variável resposta considerada foi a produtividade biológica (PB), que é relacionada à biomassa da planta.

Devido à existência de variabilidade natural entre anos agrícolas, o estudo considerou seis anos a fim de elaborar modelos confiáveis. Além disso, foi realizada uma classificação para os mesmos, 
em favoráveis, intermediários e desfavoráveis à produtividade biológica da aveia, considerando as condições meteorológicas em conjunto aos seus efeitos sobre a PB.

Para verificar diferença significativa entre os tratamentos, foi aplicada análise de variância (ANOVA), com nível de significância de $5 \%$ de probabilidade de erro [2]. Após isto, foi realizada, por regressão, a modelagem da $\mathrm{PB}$ em função da dose de $\mathrm{N}(\mathrm{DN})$ e do dia do ciclo (DC) em que foi realizado o corte de biomassa, para cada categoria de ano agrícola e também de modo geral para simulação independente de condição de ano de cultivo. O modelo de regressão por superfície de resposta pode ser representado por

$$
Z_{i}=\beta_{0}+\beta_{1} X_{i}+\beta_{2} Y_{i}+\beta_{3} X_{i}^{2}+\beta_{4} Y_{i}^{2}+\beta_{5} X_{i} Y_{i}+\varepsilon_{i}
$$

em que $Z_{i}$ é a variável dependente (PB), $\beta_{i}$ é a estimativa dos coeficientes da regressão, $X$ e $Y$ são as variáveis independentes (DN e DC) e $\varepsilon_{i}$ é o erro $[1,5]$. As análises de regressão foram realizadas através do software Genes [3].

Cabe acrescentar que em um modelo de regressão por superfície de resposta não é preciso, necessariamente, que haja todos os termos conforme equação (1). Deste modo, os modelos deste estudo foram definidos considerando os termos de regressão cujos coeficientes possuíam significância de $5 \%$ de probabilidade de erro, visando garantir termos nos modelos que, de fato, sejam relevantes no processo de modelagem. Além disto, o ajuste e a adequabilidade dos modelos também foram avaliados observando se os coeficientes de determinação $\left(R^{2}\right)$ eram adequados.

Os modelos tiveram seu desempenho analisado considerando simulações em dois momentos de corte de biomassa, sendo eles aos 90 e 120 DAE, com enfoque principal na produção de feno e silagem para alimentação animal, visto que estes são os estágios da planta em que normalmente são realizadas estas práticas [8].

\section{Resultados e Discussões}

Na classificação dos anos foram considerados fatores meteorológicos e os valores médios de produtividade biológica aos 120 DAE, apresentados na Tabela 1. Em 2013 houve temperaturas adequadas ao desenvolvimento da aveia. O fornecimento de $\mathrm{N}$ ocorreu em condições propícias de absorção pela planta, com chuvas de pouca intensidade e bem distribuídas. Neste ano, obtevese produtividade biológica média de $11637 \mathrm{~kg} \mathrm{ha}^{-1}$. Em 2015, a umidade do solo no momento da adubação nitrogenada gerou um ambiente apropriado à absorção do nutriente, aliada a temperaturas reduzidas e precipitações constantes até o florescimento, potencializando a formação e sobrevivência dos afilhos, gerando uma produtividade biológica de $11802 \mathrm{~kg} \mathrm{ha}^{-1}$. Em 2016, a ocorrência de pouco volume de chuvas, porém, de maneira bem distribuída, manteve a umidade do solo adequada, aliada a temperaturas amenas e estáveis, gerando uma produtividade de biomassa de $12096 \mathrm{~kg} \mathrm{ha}{ }^{-1}$, acima da média geral dos anos avaliados. Estas condições classificam os anos de 2013, 2015 e 2016 como favoráveis (AF) à produtividade de biomassa.

No ano de 2012, ocorreram elevadas temperaturas nos primeiros dias do ciclo que podem ter afetado a germinação da planta. Além disso, um período sem chuvas foi observado após a adubação nitrogenada, fato que reduz a absorção do nutriente, afetando a produção de afilhos. Nestas condições, a produtividade média de biomassa foi de $7528 \mathrm{~kg} \mathrm{ha}^{-1}$. No ano de 2014, a elevada precipitação durante todo o ciclo da aveia e a possível redução da radiação, afetaram o desenvolvimento de afilhos. Nestas condições, a produtividade média de biomassa foi de $7512 \mathrm{~kg}$ $h a^{-1}$. Isto classifica os anos de 2012 e 2014 como desfavoráveis (AD) à produtividade de biomassa.

Em 2011, os altos níveis de precipitação ocorridos nos dias que antecedem a adubação nitrogenada e as baixas temperaturas propiciaram condições benéficas ao desenvolvimento da planta. Contudo, longos períodos chuvosos reduziram a qualidade de radiação, fato que reduz a produção 
de afilhos. Nestas condições, a produtividade média de biomassa foi de $10844 \mathrm{~kg} \mathrm{ha}^{-1}$, classificando o ano de 2011 como intermediário (AI) à produtividade de biomassa.

Na Tabela 1, são apresentadas as médias de produtividade biológica em função da dose de nitrogênio aplicada e dia do ciclo em que é realizado o corte de biomassa, em cada ano de cultivo analisado. É possível perceber comportamento crescente da PB tanto para o aumento da dose de $\mathrm{N}$, como para o acréscimo de dias, entretanto, com influência muito mais significativa desta última variável.

Tabela 1: Médias de produtividade biológica da aveia pelas doses de nitrogênio e momento do corte de biomassa em dias após a emergência em distintos anos de cultivo.

\begin{tabular}{|c|c|c|c|c|c|c|c|c|c|c|}
\hline \multirow{2}{*}{$\begin{array}{c}\text { Dose N } \\
\left(k g h a^{-1}\right)\end{array}$} & \multicolumn{4}{|c|}{$\mathrm{PB}\left(k g h a^{-1}\right)(\operatorname{dias})$} & \multirow[t]{2}{*}{$\overline{P B}$} & \multicolumn{4}{|c|}{$\mathrm{PB}\left(k g h a^{-1}\right)(\operatorname{dias})$} & \multirow[t]{2}{*}{$\overline{P B}$} \\
\hline & 30 & 60 & 90 & 120 & & 30 & 60 & 90 & 120 & \\
\hline & \multicolumn{5}{|c|}{$2011(\mathrm{AI})$} & \multicolumn{5}{|c|}{$2012(\mathrm{AD})$} \\
\hline 0 & 487 & 1245 & 3245 & 9711 & 3672 & 247 & 1268 & 6349 & 6371 & 3559 \\
\hline 30 & 479 & 1380 & 5455 & 10390 & 4423 & 273 & 1959 & 6526 & 7030 & 3947 \\
\hline 60 & 573 & 1482 & 5571 & 11190 & 4704 & 306 & 1899 & 6869 & 7080 & 4039 \\
\hline 90 & 551 & 1528 & 5732 & 11317 & 4782 & 315 & 2156 & 7809 & 8166 & 4612 \\
\hline 120 & 544 & 1588 & 5881 & 11612 & 4906 & 330 & 2334 & 8600 & 8994 & 5065 \\
\hline \multirow[t]{2}{*}{$\overline{P B}$} & 527 & 1445 & 5177 & 10844 & - & 294 & 1923 & 7231 & 7528 & - \\
\hline & \multicolumn{5}{|c|}{$2013(\mathrm{AF})$} & \multicolumn{5}{|c|}{$2014(\mathrm{AD})$} \\
\hline 0 & 547 & 1451 & 5813 & 9920 & 4433 & 321 & 1522 & 6061 & 6164 & 3517 \\
\hline 30 & 551 & 1511 & 6954 & 11071 & 5022 & 338 & 1857 & 6359 & 6464 & 3755 \\
\hline 60 & 570 & 1538 & 6764 & 11968 & 5210 & 333 & 1924 & 6655 & 6794 & 3927 \\
\hline 90 & 575 & 1572 & 6872 & 12355 & 5344 & 349 & 2121 & 7850 & 8418 & 4685 \\
\hline 120 & 584 & 1605 & 6906 & 12870 & 5491 & 360 & 2285 & 8821 & 9719 & 5296 \\
\hline \multirow[t]{2}{*}{$\overline{P B}$} & 565 & 1535 & 6662 & 11637 & - & 340 & 1942 & 7149 & 7512 & - \\
\hline & \multicolumn{5}{|c|}{$2015(\mathrm{AF})$} & \multicolumn{5}{|c|}{$2016(\mathrm{AF})$} \\
\hline 0 & 157 & 1156 & 6191 & 9514 & 4255 & 261 & 1645 & 6998 & 9267 & 4543 \\
\hline 30 & 165 & 1811 & 6936 & 10844 & 4939 & 288 & 2404 & 8772 & 11742 & 5802 \\
\hline 60 & 181 & 2599 & 7114 & 11898 & 5448 & 321 & 2355 & 10208 & 12272 & 6289 \\
\hline 90 & 196 & 2584 & 7495 & 12877 & 5788 & 332 & 2663 & 11589 & 13135 & 6930 \\
\hline 120 & 212 & 2769 & 7826 & 13875 & 6171 & 349 & 2882 & 12984 & 14063 & 7570 \\
\hline$\overline{P B}$ & 182 & 2184 & 7112 & 11802 & - & 310 & 2390 & 10110 & 12096 & - \\
\hline
\end{tabular}

Após constatada significância dos tratamentos pela ANOVA, foi aplicada a análise de regressão por superfície de resposta, obtendo os modelos apresentados na Tabela 2. Os coeficientes de regressão foram significativos estatisticamente, com altos coeficientes de determinação, evidenciando uma modelagem significativa da produtividade biológica por categoria de ano agrícola e também de modo geral, independente de condições de ano. Analisando as regressões, verifica-se comportamento linear da PB em função da dose de nitrogênio, entretanto, quando aliada a quantidade de dias do ciclo, é possível perceber comportamento quadrático.

Nas Tabelas 3 e 4 são apresentados os valores simulados e observados de PB em função de diferentes doses de aplicação de $\mathrm{N}$ e do corte de biomassa aos 90 DAE e 120 DAE, com enfoque para a produção de feno e silagem, respectivamente. É possível verificar a comparação entre os valores reais e simulados através de erros relativos por condição de ano agrícola e também de modo 
Tabela 2: Modelos de regressão por superfície de resposta da produtividade biológica da aveia por categoria de ano em função da dose de nitrogênio e do momento de corte de biomassa.

\begin{tabular}{cccc}
\hline Categoria de ano & $P B=\beta_{0}+\beta_{1} \cdot D N+\beta_{2} \cdot D C+\beta_{3} \cdot D C^{2}$ & $\mathrm{P}$ & $R^{2}(\%)$ \\
\hline AF & $P B=-2734+15,85 D N+42,23 D C+0,62 D C^{2}$ & $*$ & 92 \\
AI & $P B=1196+9,46 D N-82,27 D C+1,32 D C^{2}$ & $*$ & 98 \\
AD & $P B=-4899+13,61 D N+143,09 D C-0,36 D C^{2}$ & $*$ & 88 \\
\hline Geral & $P B=-2801+14,04 D N+55,10 D C+0,41 D C^{2}$ & $*$ & 86
\end{tabular}

$\mathrm{PB}=$ produtividade biológica; $\mathrm{DN}=$ dose de nitrogênio; $\mathrm{DC}=$ dias do ciclo após a emergência no momento de corte de biomassa; $\mathrm{AF}=$ ano favorável; $\mathrm{AD}=$ ano desfavorável; $\mathrm{AI}=$ ano intermediário; $\mathrm{P}=$ probabilidade dos coeficientes de regressão; $*$ significativo a $5 \%$ de probabilidade de erro, pelo teste t; $R^{2}=$ coeficiente de determinação.

geral. Na Tabela 3, em anos favoráveis à produtividade de biomassa, o modelo apresentou erros relativos entre 3,8\% e 13,5\%. Nos anos intermediários, foram observados erros entre 4,4\% e $38,1 \%$. Em anos desfavoráveis, houve erros entre $13 \%$ e 23,1\%. Na análise do modelo geral, independente de condição de ano, foram constatados erros entre $5,1 \%$ e $15,7 \%$. Cabe acrescentar que todas as magnitudes mais elevadas nos erros foram observadas em situações extremas de aplicação de N, como a dose nula e a dose de $120 \mathrm{~kg} \mathrm{ha}^{-1}$, que são práticas normalmente não adotadas pelos produtores rurais. Assim, de modo geral, os modelos apresentaram bom desempenho na simulação da produtividade biológica.

Tabela 3: Comparação da produtividade biológica de aveia observada e simulada com corte de biomassa aos 90 dias do ciclo por categoria de ano em função de diferentes doses de nitrogênio.

\begin{tabular}{|c|c|c|c|c|c|c|}
\hline \multirow[t]{2}{*}{ Categoria de ano } & \multirow[t]{2}{*}{ Condição } & \multicolumn{5}{|c|}{ Doses de nitrogênio $\left(k g h a^{-1}\right)$} \\
\hline & & 0 & 30 & 60 & 90 & 120 \\
\hline \multirow{4}{*}{$\mathrm{AF}$} & PB observada & 6334 & 7554 & 8029 & 8652 & 9239 \\
\hline & & $P B=$ & -273 & +15, & $5 D N$ & $C+0,62 D C^{2}$ \\
\hline & PB simulada & 6089 & 6564 & 7040 & 7515 & 7991 \\
\hline & Erro relativo (\%) & 3,8 & 13,1 & 12,3 & 13,1 & 13,5 \\
\hline \multirow{4}{*}{$\mathrm{AI}$} & PB observada & 3245 & 5455 & 5571 & 5732 & 5881 \\
\hline & & $P B$ & $=1196$ & $+9,46$ & $D N-$ & $+1,32 D C^{2}$ \\
\hline & PB simulada & 4484 & 4768 & 5051 & 5335 & 5619 \\
\hline & Erro relativo (\%) & 38,1 & 12,6 & 9,3 & 6,9 & 4,4 \\
\hline \multirow{4}{*}{$\mathrm{AD}$} & PB observada & 6205 & 6442 & 6762 & 7830 & 8710 \\
\hline & & $P B=$ & -4899 & $+13,6$ & $D N+$ & $C-0,36 D C^{2}$ \\
\hline & PB simulada & 5063 & 5471 & 5880 & 6288 & 6696 \\
\hline & Erro relativo (\%) & 18,4 & 15,0 & 13,0 & 19,7 & 23,1 \\
\hline \multirow{4}{*}{ Geral } & PB observada & 5776 & 6834 & 7197 & 7891 & 8503 \\
\hline & & $P B=$ & -280 & +14, & $4 D N$ & $C+0,41 D C^{2}$ \\
\hline & PB simulada & 5479 & 5900 & 6321 & 6743 & 7164 \\
\hline & Erro relativo (\%) & 5,1 & 13,7 & 12,2 & 14,5 & 15,7 \\
\hline
\end{tabular}

$\overline{\mathrm{PB}}=$ produtividade biológica; $\mathrm{DN}=$ dose de nitrogênio; $\mathrm{DC}=$ dias do ciclo após a emergência no momento de corte de biomassa; $\mathrm{AF}=$ ano favorável; $\mathrm{AD}=$ ano desfavorável; $\mathrm{AI}=$ ano intermediário.

Na Tabela 4, dos dados referentes ao corte de biomassa aos 120 DAE, foram encontrados erros mais reduzidos em comparação aos da Tabela 3, de corte aos 90 DAE. Em anos favoráveis à produtividade biológica, os erros variaram entre $0,8 \%$ e 17,7\%. Nos anos intermediários, ficaram entre $1,2 \%$ e $6,4 \%$. E em anos desfavoráveis, entre $0,3 \%$ e 13,9\%. No modelo geral, independente de condição de ano, os erros evidenciaram valores entre $0,6 \%$ e 14,4\%. Assim como nos erros 
relacionados ao corte aos 90 DAE, a maioria dos erros relativos da Tabela 4 com maiores magnitudes ocorrem em dose de $\mathrm{N}$ normalmente não praticada por produtores rurais (neste caso, a dose nula). Isto evidencia bom desempenho dos modelos encontrados para a simulação de PB, quando aplicados manejos comuns de cultivo.

Tabela 4: Comparação da produtividade biológica de aveia observada e simulada com corte de biomassa aos 120 dias do ciclo por categoria de ano em função de diferentes doses de nitrogênio.

\begin{tabular}{|c|c|c|c|c|c|c|}
\hline \multirow[t]{2}{*}{ Categoria de ano } & \multirow[t]{2}{*}{ Condição } & \multicolumn{5}{|c|}{ Doses de nitrogênio $\left(k g h a^{-1}\right)$} \\
\hline & & 0 & 30 & 60 & 90 & 120 \\
\hline \multirow{4}{*}{$\mathrm{AF}$} & PB observada & 9567 & 11219 & 12046 & 12789 & 13603 \\
\hline & & $P B=$ & -2734 & $15,85 L$ & $N+42$, & $+0,62 D C^{2}$ \\
\hline & PB simulada & 11262 & 11737 & 12213 & 12688 & 13164 \\
\hline & Erro relativo $(\%)$ & 17,7 & 4,6 & 1,4 & 0,8 & 3,2 \\
\hline \multirow{4}{*}{$\mathrm{AI}$} & PB observada & 9711 & 10390 & 11190 & 11317 & 11612 \\
\hline & & $P B$ & $=1196+$ & $9,46 D I$ & $-82,2$ & $-1,32 D C^{2}$ \\
\hline & PB simulada & 10332 & 10615 & 10899 & 11183 & 11467 \\
\hline & Erro relativo $(\%)$ & 6,4 & 2,2 & 2,6 & 1,2 & 1,2 \\
\hline \multirow{4}{*}{$\mathrm{AD}$} & PB observada & 6268 & 6747 & 6937 & 8292 & 9357 \\
\hline & & $P B=$ & $-4899+$ & $13,61 D$ & $V+143$ & $-0,36 D C^{2}$ \\
\hline & PB simulada & 7088 & 7496 & 7904 & 8313 & 8710 \\
\hline & Erro relativo (\%) & 13,1 & 11,1 & 13,9 & 0,3 & 6,9 \\
\hline \multirow{4}{*}{ Geral } & PB observada & 8491 & 9590 & 10200 & 11045 & 11855 \\
\hline & & $P B=$ & -2801 & $14,04 L$ & $N+55$ & $+0,41 D C^{2}$ \\
\hline & PB simulada & 9715 & 10136 & 10557 & 10979 & 11400 \\
\hline & Erro relativo (\%) & 14,4 & 5,7 & 3,5 & 0,6 & 3,8 \\
\hline
\end{tabular}
biomassa; $\mathrm{AF}=$ ano favorável; $\mathrm{AD}=$ ano desfavorável; $\mathrm{AI}=$ ano intermediário. 


\section{Conclusões}

Os modelos de regressão obtidos por superfície de resposta evidenciam eficiência na simulação da $\mathrm{PB}$ em situações reais de cultivo da aveia, com possibilidade de estimativa de PB em função da dose de $\mathrm{N}$ e do momento de corte de biomassa, com finalidade na produção de feno e silagem. Os modelos obtidos para simulação, em condições de ano favoráveis à produtividade biológica, em condições intermediárias, em condições desfavoráveis, e em situações independentes de condição de ano, apresentam erros relativos adequados em cenários normalmente praticados por produtores rurais, evidenciando, assim, sua recomendação.

\section{Agradecimentos}

À CAPES, ao CNPq, à FAPERGS e à UNIJUÍ pelos recursos financeiros e estrutura física e material necessários para realização da pesquisa.

\section{Referências}

[1] Almeida, R. L. da S., Almeida Filho, R. L. da S., Silva, R. L. e Almeida, G. V. de M. A. Maximização da produção do cacaueiro CCN-51 através de modelos de regressão, Revista Brasileira de Agricultura Irrigada, 12:2845-2853, 2018. DOI: 10.7127/rbai.v12n500837.

[2] Coelho, A. P., De Faria, R. T., Dalri, A. B., Palaretti, L. F. e Zanini, J. R. Clorofilômetro portátil como forma de manejo da irrigação e adubação nitrogenada em aveia-branca, Revista Brasileira de Agricultura Irrigada, 12:2542-2553, 2018. DOI: 10.7127/rbai.v12n200803.

[3] Cruz, C. D. Programa GENES: estatística experimental e matrizes. UFV, Viçosa, 2006.

[4] Da Silva, R. W. V., Martins, G. M. G., Do Nascimento, R. A., Viana, A. F. da S., De Aguiar, F. S. e Da Silva, B. A. Uso da metodologia de superfície de resposta na otimização da extração de compostos fenólicos da casca dos frutos de Hymenaea courbaril L. (Jatobá), Brazilian Journal of Food Technology, 22:1-13, 2019. DOI: 10.1590/1981-6723.08918.

[5] Kraisig, A. R., Scremin, O. B., Mantai, R. D., Marolli, A., De Mamann, A. T. W., Brezolin, A. P., Alessi, O. e Da Silva, J. A. G. Regressão por superfície de resposta pelo uso combinado de nitrogênio e hidrogel no sistema milho/aveia, Proceeding Series of the Brazilian Society of Computational and Applied Mathematics, volume 6, 2018. DOI: 10.5540/03.2018.006.02.0437.

[6] Ma, B. L., Zheng, Z., Pageau, D., Vera, C., Fregeau-Reid, J., Xue, A. and Yan, W. Nitrogen and phosphorus uptake, yield and agronomic traits of oat cultivars as affected by fertilizer $\mathrm{N}$ rates under diverse environments, Nutrient Cycling in Agroecosystems, 108:245-265, 2017. DOI: $10.1007 / \mathrm{s} 10705-017-9848-8$.

[7] Marolli, A., Da Silva, J.A.G., Sawicki, S., Binelo, M.O., Scremin, A.H., Reginatto, D.C., Dornelles, E.F. e Lambrecht, D.M. A simulação da biomassa de aveia por elementos climáticos, nitrogênio e regulador de crescimento, Arquivo Brasileiro de Medicina Veterinária e Zootecnia, 70:535-544, 2018. DOI: 10.1590/1678-4162-9504.

[8] Moreira, G. L. P., Moreira, E. de S., Prates, C. J. N., Cardoso Júnior, N. dos S., Viana, A. E. S. e Lopes, S. C. Produtividade de biomassa e composição bromatológica de genótipos de aveia forrageira em Vitória da Conquista, BA, Scientia Plena, 13:1-10, 2017. DOI: $10.14808 /$ sci.plena.2017.030201. 